\title{
Study by laser interferometry of the resonance modes of the shear plate used in the Standards characterization of piezoceramics
}

\author{
L. Pardo ${ }^{1}$, F. Montero de Espinosa ${ }^{2}$ and K. Brebøl ${ }^{3}$ \\ ${ }^{1}$ Instituto de Ciencia de Materiales de Madrid (ICMM). CSIC. Cantoblanco 28049-Madrid \\ (Spain) \\ ${ }^{2}$ Instituto de Acústica. CETEF. CSIC. Serrano, 144. 28006-Madrid (Spain) \\ ${ }^{3}$ Limiel ApS. Langebæk. (Denmark)
}

\begin{abstract}
Recent Finite Element Analysis (FEA) modeling of a Standard shear plate of Navy II type PZT-based ceramics was carried out based on the full matrix material characterization Material coefficients in the linear range, including all losses, were obtained by an iterative automatic method from impedance measurements at resonance. FEA modeling has shown that the shear plate used in the standard characterization of piezoceramics is dynamically clamped at resonance, which results in an underestimation of the related shear coefficients.

This work aims to examine experimentally the resonance modes of such standard shear sample by the use of the laser interferometry technique to map the displacement pattern at its free surfaces.
\end{abstract}

Keywords: Laser interferometry, Shear resonance, PZT, Material characterization, Standards 


\section{Introduction}

Electromechanical characterization in the linear range of ferro-piezoelectric bulk ceramic materials from impedance measurements at resonance has been used since early times of the development of these materials in the 60's [1], and become a widespread practice. The simple experimental set-up, involving an impedance analyzer and appropriated sample holder has contributed to such spreading and the standardization of the technique [2,3].

The limitations of the Standards methods, generally accepted, on the characterization of materials with high losses and low electromechanical coefficients, were lately focused by several authors. The development of iterative methods $[1,4,5]$ for to solve the analytical expression of the wave equation related to a given resonance brought the possibility to determine complex material coefficients from such measurements, thus accounting for the dielectric, mechanical and piezoelectric losses of the material.

Nowadays, the systematic application of one of these, Alemany et al. automatic iterative method [4,5], to all geometries needed, allowed the complete characterization of a commercially available Navy II type PZT-based commercial ceramic, that involves the determination of ten piezoelectric, elastic and dielectric coefficients [6]. To do so, and according to Standards [2, 3], measurements at four resonance modes of three sample shapes (Figure 1) are enough: the thickness and radial mode of thin disks, thickness poled, the length extensional mode of long rods or bars, length poled, and the thickness shear mode of a shear plate. Such Standard shear plate is poled along one of the two plate sides. Then poling electrodes are removed and new ones are attached to the two major faces of the plate for the measurement. For this Standard shear sample the appearance of satellite peaks at both sides of the main shear resonance is currently observed [6] and often related to sample inhomogeneities. It is also known that the piezoelectric shear coefficients measured using this Standard shear sample are underestimated [7]. Based on the full characterization mentioned above, FEA modeling has been used both to test the validity of the matrix of coefficients obtained by Alemany et al. method by reproduction of the measured spectra of the samples used in the characterization [7], and to understand the modes of vibration of the ceramic resonators involved in such characterization, in particular the Standard shear plate [8], in order to improve the characterization and to get more accurate material properties.

This work aims to obtain experimental evidence on the modes of vibration of the Standard shear plate geometry by measurement of the normal displacement at the free surfaces of the sample by a laser interferometry technique. The results here obtained by interferometry are compared with the predictions of the previous FEA modeling of this sample [8]. 


\section{Experimental}

A shear plate of a Navy II type PZT-based commercial ceramic (PZ27 from Ferroperm Piezoceramics A/S) fulfilling Standards relation among dimensions, L,w $>10 t$ (Figure 1), and actual dimensions of $0.59 \mathrm{~mm}$ thickness, t, $5.9 \mathrm{~mm}$ width for poling, w, and $5.9 \mathrm{~mm}$ length, $\mathrm{L}$, was here studied. Another sample with dimensional ratios close to the Standard, and with actual dimensions 2x15x15 mm, was also studied to improve the experiment resolution. Differences among them were not found either in the main features of the resonance spectra obtained by impedance measurements or in those of the interferometry measurements.

The experiment set-up for the normal displacement to the free sample surfaces measurement at resonance consists of a computer controlled 2D stage (Microcontrole T64) with $10 \mu \mathrm{m}$ precision and a laser vibrometer Polytec OFV-505, similar to the one described elsewhere [9]. The sample is gently placed on a rigid block to avoid mechanical constraints. The piezoceramic plate is excited at the resonances with a Signal Generator HP 8116A. Electrical connection is provided by contact to the plate electrodes using thin cooper wires $-0.1 \mathrm{~mm}$ thick -, which are also used as fixture. Long burses are used to arrive to stationary response. The plate performs a 2D movement acquiring the vibration signal at each scan step. The signal from the vibrometer is gated and peak detected using a Panametric 5052UA Ultrasonic Analyzer. Finally, a computer program presents the scan of the surfaces as an image where the color or the gray level is proportional to the amplitude displacement perpendicular to the scanned surface.

\section{Results}

The R and G peaks at the resonance spectra of a PZ27 Standard shear plate of 2x15x15mm are shown in Figure 2, where the appearance of satellite peaks is observed. Figures 3 and 4 show the normal displacement to the scanned surfaces of the same plate for the frequencies corresponding to the main shear resonance, at $444 \mathrm{kHz}$, and one of the satellite peaks, at $380 \mathrm{kHz}$.

The scan in Figure 3(a) shows normal displacement with a clear pattern of interference between two standing waves in perpendicular directions along the plate. A thin line crossing the image at the upper right corner corresponds to the light reflected by a copper wire used to hold the sample.

Figure 3(b), which corresponds to a horizontal side, at the top of Figure 3(a), shows very little displacement normal to the surface. However, Figure 3(c), that correspond to a vertical side, at the left of Figure 3(a), shows a central line almost immobile (dark) with bands of increasing normal displacement (towards brighter grey level) symmetrically located up and down 
of this line, as it corresponds to a shear movement at the side of the plate. Superimposed to this shear movement at the vertical side, there is a periodic normal displacement in areas close to the edges of this side. Therefore, this is a complex mode of motion.

The scan at $444 \mathrm{kHz}$ at the major surface of the plate, Figure 4(a), shows a butterfly-like pattern of interference, in which it is remarkable that the normal displacement at the center of the plate is higher that at the horizontal sides, top and bottom of the image and, overall, the normal displacement is smaller than at $380 \mathrm{kHz}$. However, as expected, the normal displacement is not null, as there is contribution of a plane wave and as the shear movement does not have a pure inplane displacement.

Additionally, Figure 4(c) shows a much more clear bands of normal displacement than Figure 4(c), corresponding to a less disturbed shear movement. Also this scan show an overall much lower normal displacement at the edges of this side. Figure 4(b) was obtained with an external amplification to get a comparable scan and shows the lowest normal displacement among all scans.

\section{Discussion}

The main peaks of this shear plates are always accompanied by satellite ones (Figure 1), which is often attributed to material inhomogeneity, such as that due to inhomogeneous poling caused by edge effects. Such explanation is doubtful, since satellite peaks are reproduced when a dielectrically, mechanically and piezoelectrically homogeneous item of PZ27 is modeled by FEA [8], and thus satellites must be understood as natural resonances associated to the geometry and dimensional ratios of the Standard shear plate. The results here presented are interesting to understand the complex modes of motion in these resonances.

FEA modeling results [8] show that the mode of motion corresponding to the satellite resonance taking place at lower frequency (here at $330 \mathrm{kHz}$ ) than the fundamental shear resonance is a complex one. It can be described by a composition of plate waves in perpendicular directions, an asymmetrical Lamb and a Love wave types, superimposed to a shear wave.

The type of normal displacement to the surface, shown in the scan of Figure 3(c), confirms the shear movement at 330kHz. The distortion at the edges of such shear in Figure 3(c), together with the pattern of interference that Figure 3(a) shows, confirms, also, that the plate at the same time experiments a composition of plate waves.

FEA modeling of the mode of motion at the fundamental shear resonance (here at $444 \mathrm{kHz}$ ) shows also [8] that it is much closer to a pure shear resonance and that there is a much less movement at the edges than there is at the center of the plate. 
As already stated, Figure 4(a) and Figure 4(c) shows less distortion from a pure shear movement for the resonance at $444 \mathrm{kHz}$ than the one observed at $380 \mathrm{kHz}$. Figure 4(b) shows the smallest normal displacement of all sides measured and Figure 4(c) shows smaller displacement than Figure 3(c), thus the edges show comparatively less movement for $444 \mathrm{kHz}$ than for $380 \mathrm{kHz}$. Also Figure 4(a) shows higher displacement in the center than near the edges, in particular than at the top and bottom sides.

Results are consistent with the FEA modeling based on the full matrix characterization of the material by an iterative automatic method from impedance measurements at resonance [8], and confirm the dynamical clamping at the main shear resonance predicted by FEA modeling.

\section{Conclusions}

It was presented here experimental evidence of the modes of motion of the Standard shear plate near the fundamental shear mode of Navy II type PZT commercial piezoceramic. The patterns of interference obtained in the measurement of the normal displacement at the major surfaces show that the shear modes of motion are in fact composition of two plate waves, asymmetric Lamb waves and/or Love waves, in addition to the shear wave.

Besides, the normal displacement at the major faces of the sample also provides information on the shear movement, occurring mainly in-plane, but, due to a non-zero Poisson ratio in the material, also showing a component normal to the surface. Interferometry results presented here show that, at the main shear resonance mode, the shear displacement takes place inhomogeneously, with a butterfly-like pattern, and it is lower at the edges of the plate. Also, scans at the sides of the shear element shows that they undergoes the lowest normal displacements of all those measured.

\section{Acknowledgements}

This work has benefited from the synergy provided by the POlar ELEtroCERamics, POLECER (G5RT-CT2001-05024) Thematic Network of the EC GROWTH Program under the $5^{\text {th }}$ RTD Framework Program. Authors want to thank Dr. Erling Ringaard from Ferroperm Piezoceramics A/S for providing shear samples and Mr. A. García, holder of a Polecer linked grant at ICMM-CSIC, for his help with the impedance measurements. L. Pardo acknowledges the financial support of project MAT-2004-0868 of the Spanish MEC. 


\section{References}

[1]L. Pardo, C. Alemany, J. Ricote, A. Moure, R. Poyato and M. Algueró. Proc. of Internacional Conference on Technology and Design of Integrated Piezoelectric Devices, A POLECER Symposium, 2-4 February, 2004. Courmayeur (Italy). pp 145-158.

[2] “IEEE Standard on piezoelectricity”. ANSI/IEEE Std. 176-1987.

[3] "Piezoelectric properties of ceramic materials and components. Part 2: methods of measurement - Low power” European Standard CENELEC, EN 50324-2.

[4] C. Alemany, L. Pardo, B. Jiménez, F. Carmona, J. Mendiola and A.M. González. J. Phys. D: Appl. Phys. 27, 148 (1994).

[5] C. Alemany, A.M. González, L. Pardo, B. Jiménez, F. Carmona and J. Mendiola. J. Phys. D: Appl.Phys. 28(5), 945 (1995).

[6] M. Algueró, C. Alemany, L. Pardo and A. M. Gonzalez. J. Am. Ceram. Soc. 87(2), 209 (2004).

[7] L. Pardo, M. Algueró and K. Brebøl . Ferroelectrics 336 , 181-190 (2006).

[8] L. Pardo, M. Algueró and K. Brebøl. Journal de Physique IV France 128, 207-211(2005).

[9] F.J. Chinchurreta, F. Montero de Espinosa. Bol.Soc.Esp.Ceram.Vid. 41(1), 71-74(2002). 


\section{Figure captions}

Figure 1. The three piezoceramic sample Standard geometries needed for the determination of the full matrix of coefficients for a $6 \mathrm{~mm}$ symmetry material: (a) thin disks, thickness poled, (b) shear plates and (c) long bars, length poled. $\mathrm{P}=$ sample polarization.

Figure 2. Resistance (R) and conductance $(G)$ for the fundamental shear thickness resonance of a thickness plate of PZ27. The symbols represent the experimental data and the lines are the reconstructed spectra of the main resonance after the values of the parameters determined by Alemany t al. method.

Figure 3. Displacements scan at $330 \mathrm{kHz}$ for: (a) the major surface of the shear plate, (b) the lateral horizontal surface of the plate and (c) the lateral vertical surface. The $\mathrm{x}$ and $\mathrm{y}$-axes indicates the number of motor steps for the scan. Step for the (a) scan is $0.1 \mathrm{~mm}$, whereas for (b) and (c) is $0.02 \mathrm{~mm}$. The color code indicates the displacement perpendicular to the scanned surface in a.u. /1V driving voltage.

Figure 4. Displacements scan at $444 \mathrm{kHz}$ for: (a) the major surface of the shear plate, (b) the lateral horizontal surface of the plate and (c) the lateral vertical surface. The $\mathrm{x}$ and $\mathrm{y}$-axes indicates the number of motor steps for the scan. Step for the (a) scan is $0.1 \mathrm{~mm}$, whereas for (b) and (c) is $0.02 \mathrm{~mm}$. The color code indicates the displacement perpendicular to the scanned surface in a.u. $/ 1 \mathrm{~V}$ driving voltage. 
(a)

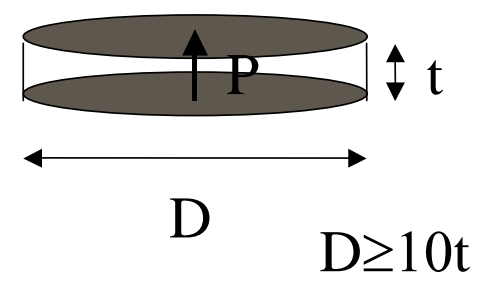

(b)

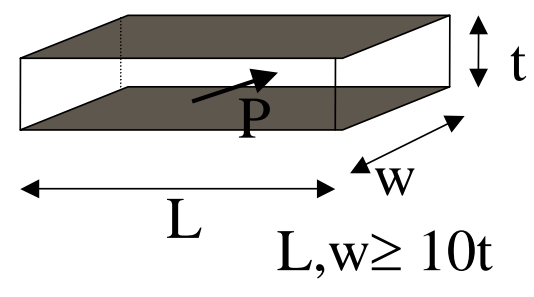

(c)

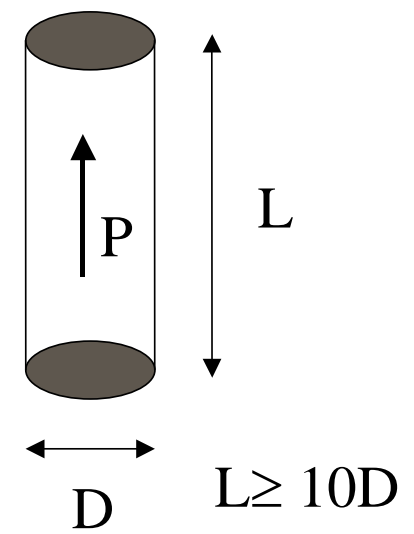

Figure 1. The three piezoceramic sample Standard geometries needed for the determination of the full matrix of coefficients for a $6 \mathrm{~mm}$ symmetry material: (a) thin disks, thickness poled, (b) shear plates and (c) long bars, length poled. $\mathrm{P}=$ sample polarization. 
PZ27 Standard shear element 15×15×2 mm

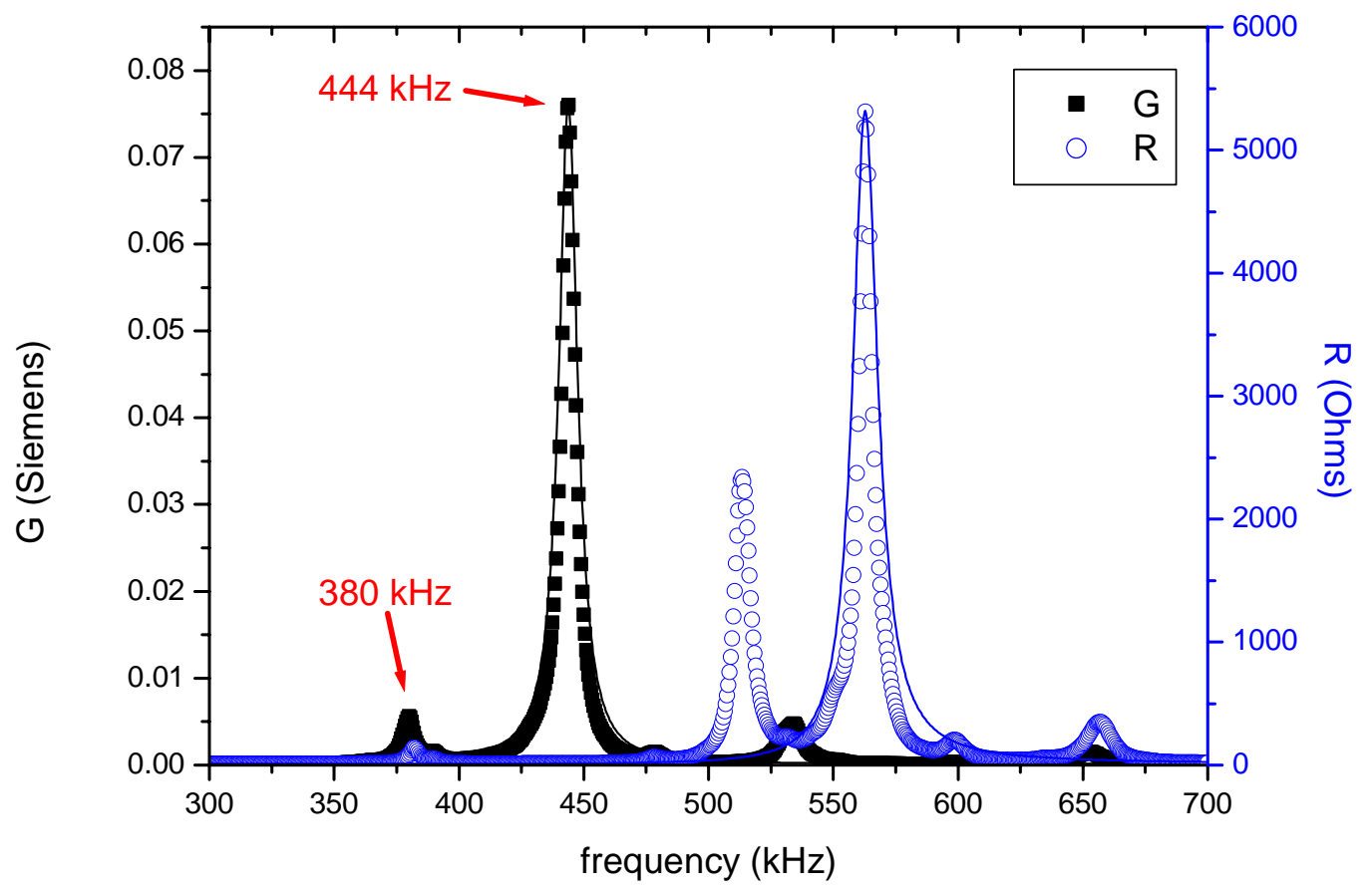

Figure 2. Resistance (R) and conductance $(G)$ for the fundamental shear thickness resonance of a thickness plate of PZ27. The symbols represent the experimental data and the lines are the reconstructed spectra of the main resonance after the values of the parameters determined by Alemany $t$ al. method. 


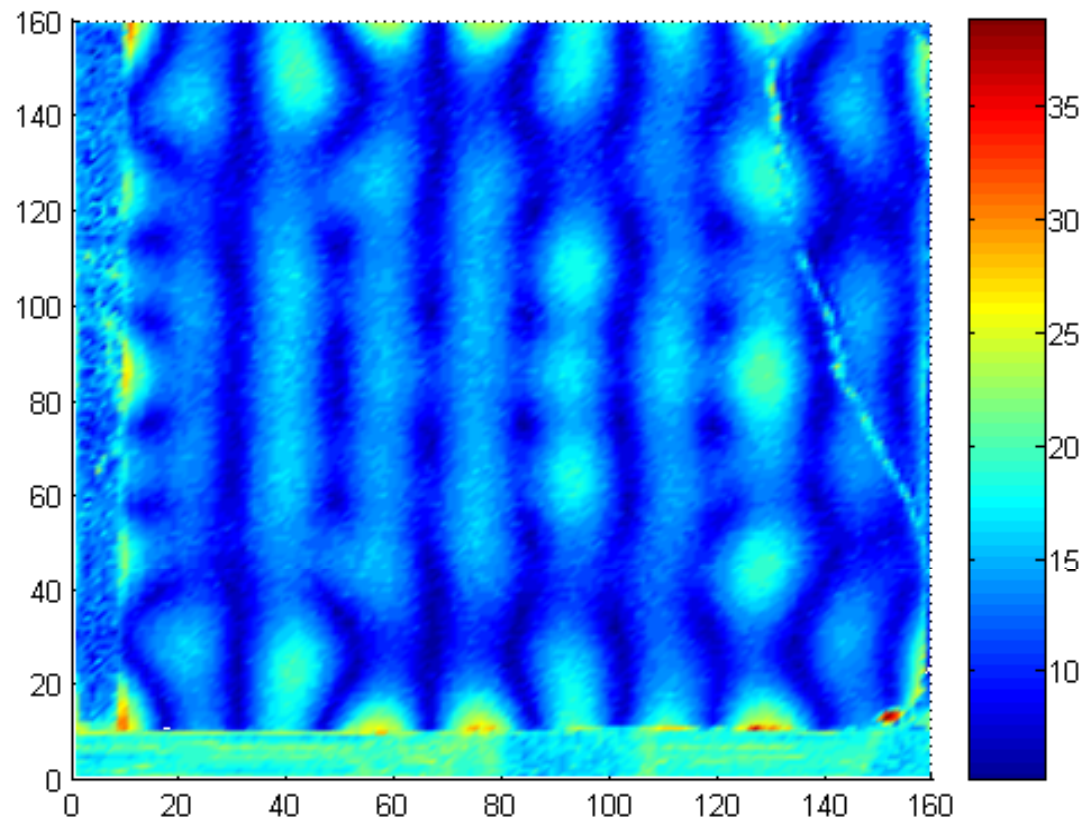

(a)

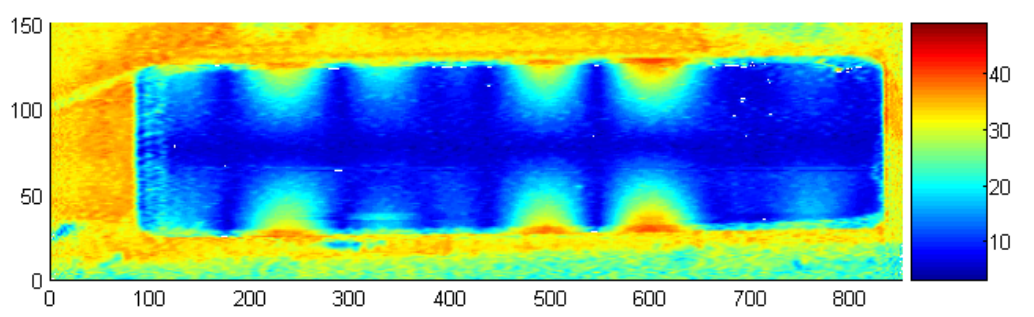

(b)

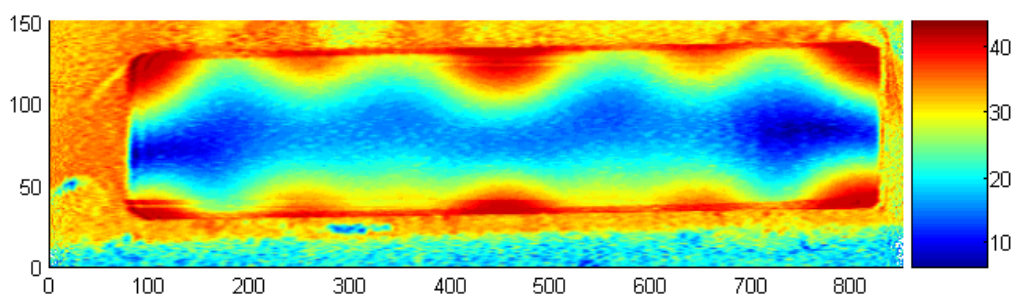

(c)

Figure 3. Displacements scan at $330 \mathrm{kHz}$ for: (a) the major surface of the shear plate, (b) the lateral horizontal surface of the plate and (c) the lateral vertical surface.

The $x$ and $y$-axes indicates the number of motor steps for the scan. Step for the (a) scan is $0.1 \mathrm{~mm}$, whereas for (b) and (c) is $0.02 \mathrm{~mm}$. The color code indicates the displacement perpendicular to the scanned surface in a.u. $/ 1 \mathrm{~V}$ driving voltage. 


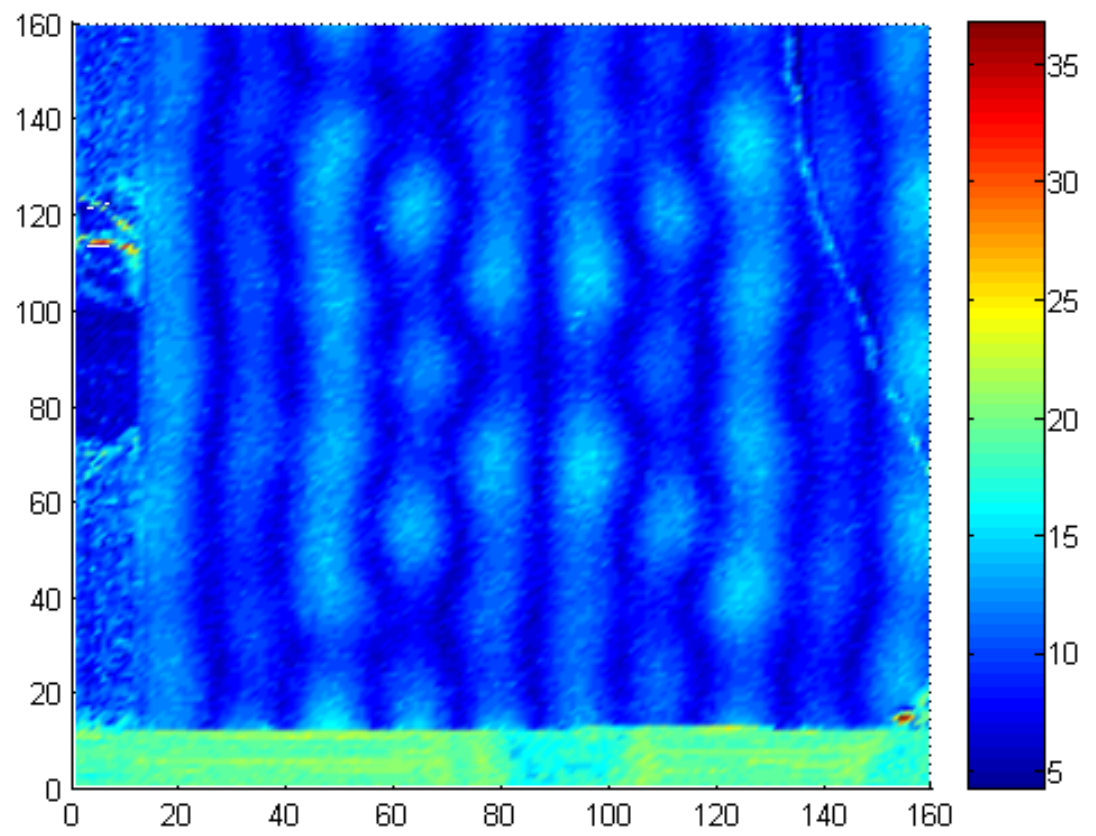

(a)
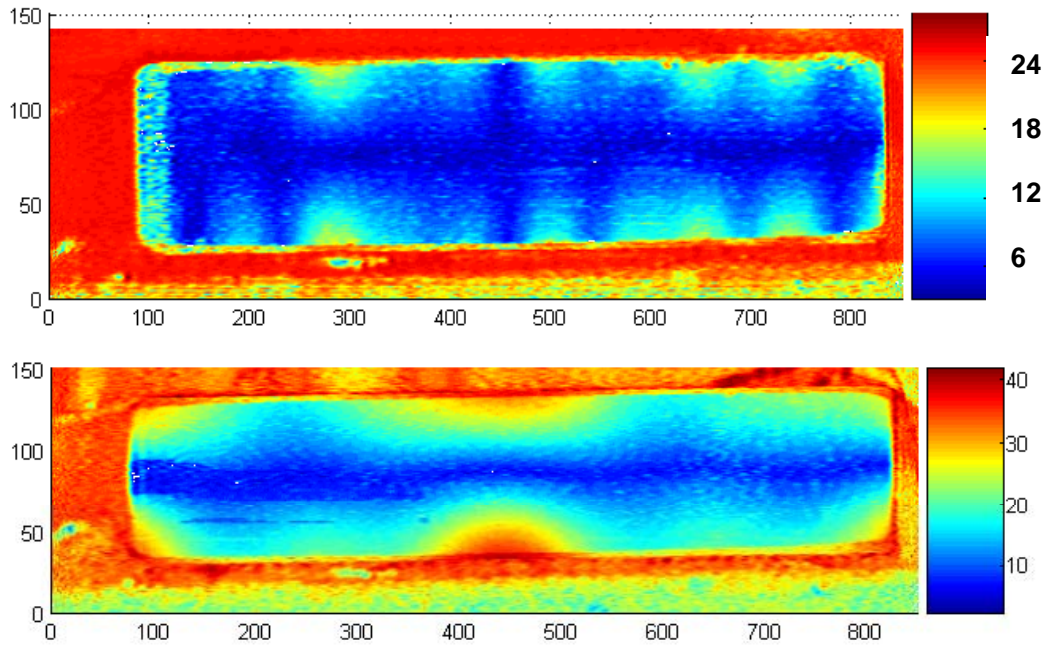

(b)

(c)

Figure 4. Displacement scans at $444 \mathrm{kHz}$ for: (a) the major surface of the shear plate, (b) the lateral horizontal surface of the plate and (c) the lateral vertical surface. The $x$ and $y-$ axes indicates the number of motor steps for the scan. Step for the (a) scan is $0.1 \mathrm{~mm}$, whereas for (b) and (c) is $0.02 \mathrm{~mm}$. The color code indicates the displacement perpendicular to the scanned surface in a.u. /1V driving voltage. 